\title{
Applying Integrated Grey System Theory and Sensor Technology to Study Influence of Cutting Conditions on Thermal Error Modeling of Machine Tools
}

\author{
Kun-Chieh Wang, Chi-Hsin Yang, ${ }^{*}$ Long Wu, and Zijian Ai \\ School of Mechanical and Electric Engineering, Sanming University, \\ Sanming City, Fujian Province 365004, China
}

(Received June 30, 2020; accepted November 24, 2020)

Keywords: displacement sensors, temperature sensors, thermal error modeling, machine tools, grey system theory, artificial neural network

To produce a good machine tool, the thermally induced error in the machine during machining plays a crucial role and is an important issue needing to be resolved. The thermal error may account for $70 \%$ of the total error. There are three main approaches to solving the thermal error problem: preventing heat flows from hot components, designing a thermally stable structure for the machine, and compensating the thermal error using thermal error models. The first two approaches can be carried out in the primary design stage of machine tools, and they have been used in the manufacture of commercial products. The third approach, the strategy of thermal error compensation, is the most effective and popular approach. However, there are still many unsolved problems. Among these problems, the cutting conditions have a significant influence on the modeling precision of the thermal error. In this study, we develop an integral model based on the integrated grey system theory (IGST) in conjunction with a genetic-algorithm (GA)-optimized back-propagation neural network (BPNN) to investigate the influence of cutting conditions on a machine tool's thermal error. The model is chosen on account of its high ability in dealing with a small amount of training data. Results show that a single thermal error modeling formula cannot make accurate predictions for different cutting conditions. Suitable adjustment of the modeling parameters or the use of a multiple modeling scheme is needed.

\section{Introduction}

Machining accuracy is the most important goal of machine tool producers. Among the errors occurring in machining, such as the vibration error, thermal error, kinematic error, tool deformation error, and wearing error between moving components, the thermal error, induced by deformation due to the temperature variation, is the primary cause of machining inaccuracy. The thermal error may account for over $70 \%$ of the total error. ${ }^{(1)}$ From careful observations, there are two types of heat sources in machining, called internal and external heat sources. 
The internal heat sources include heat generated in servo motors, metal cutting processes, and various frictions arising from motion in mechanisms such as spindles, bearings, ball screws, belts, gears, and guideways. The external heat sources include room temperature changes, solar radiation, and human bodies. To reduce the thermal error, three main approaches are usually used: avoidance of thermal deformation, control of the heat flow, and compensation of thermal error. $^{(2)}$ Among these methods, thermal error compensation is not only easy but also efficient. ${ }^{(3-20)}$ Thus, it is the most commonly used way of enhancing the machining accuracy of machine tools. Generally speaking, a thermal error compensation procedure includes the following steps. First, suitable experimental conditions are set in order to simulate the machining conditions of the target machine. Second, characteristic points on the machine are determined for temperature measurements. Third, an appropriate integrated measuring system is set up, then the deformation of the tool tip and the temperature at the chosen characteristic points are simultaneously detected. Fourth, a robust thermal error model is built that well describes the relationship between temperature rises and thermal displacements. Fifth, the established thermal error model is verified via experiments.

Despite the issues of thermal error compensation of machine tools, which have been studied for a long time, there still remain some unsolved problems, such as how to establish a robust and precise thermal error model for different kinds of machines. One of the key issues that determine the effectiveness of a thermal error model is the machining conditions in modeling experiments. Many past studies considered only the thermal deformation of the spindle since it is the main component that is deformed in machine tools. Therefore, only the spindle rotational velocity was considered as the parameter in setting the experimental cutting conditions. ${ }^{(2,10,11)}$ However, thermal deformation may occur in any component of a machine. Thus, more parameters should be considered when setting experimental cutting conditions. Raja et al. ${ }^{(7)}$ and Wang et al. ${ }^{(9)}$ adopted the parameters of spindle rotational velocity and feeding speed in setting experimental cutting conditions. Note that no previous studies have systematically considered the effect of changing the parameters that influence the cutting conditions.

Different machining conditions usually generate different amounts of heat and therefore result in different temperature distributions and thermal displacements in the machine structure. Machining conditions significantly influence the method of building a thermal error model as well as its prediction ability. To address the aforementioned matters, in this study, we investigate the influence of different machining conditions on the thermal behavior of a machine structure and build a thermal error model. A computer numerical control (CNC) vertical machining center is chosen as the investigation target (made by Taiwan's Litz machinery company, model DV-1000), as illustrated in Fig. 1.

\section{Analysis of Machining Conditions}

In general, the machining conditions of a $\mathrm{CNC}$ machine tool are determined by the shape and material of the work pieces and its functions as well as capabilities (such as the maximum rotational speed of the spindle and the linear feeding speed). In machining experiments toward building a thermal error model, the following variations of parameters are usually taken into 


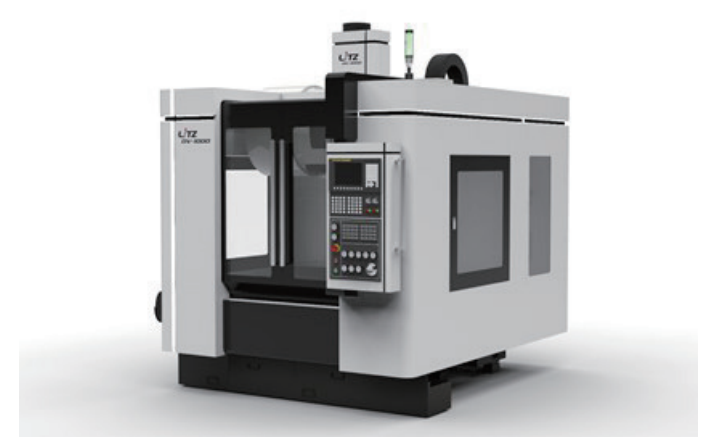

Fig. 1. (Color online) Target $\mathrm{CNC}$ vertical machining center.

Table 1

Non-periodic soft and periodic hard machining conditions.

\begin{tabular}{lcccc}
\hline$S$ & 1st section & 2nd section & 3rd section & 4th section \\
$F$ & 0-30 min & 30-60 min & 60-90 min & 90-120 min \\
\hline \multicolumn{5}{c}{ Non-periodic cutting case } \\
\hline Soft cutting & $S: 1000 \mathrm{rpm}$ & $S: 2000 \mathrm{rpm}$ & $S: 3000 \mathrm{rpm}$ & $S: 4000 \mathrm{rpm}$ \\
(Aluminum) & $F: 10 \mathrm{~m} / \mathrm{min}$ & $F: 20 \mathrm{~m} / \mathrm{min}$ & $F: 30 \mathrm{~m} / \mathrm{min}$ & $F: 40 \mathrm{~m} / \mathrm{min}$ \\
\hline \multicolumn{5}{c}{ Periodic cutting case } \\
\hline Hard cutting & $S: 2000 \mathrm{rpm}$ & $S: 4000 \mathrm{rpm}$ & $S: 2000 \mathrm{rpm}$ & $S: 4000 \mathrm{rpm}$ \\
(SC45) & $F: 20 \mathrm{~m} / \mathrm{min}$ & $F: 40 \mathrm{~m} / \mathrm{min}$ & $F: 20 \mathrm{~m} / \mathrm{min}$ & $F: 40 \mathrm{~m} / \mathrm{min}$ \\
\hline
\end{tabular}

$S$ : Spindle speed, $F$ : Feeding speed along $X, Y$, and $Z$ axes

consideration: (1) different machining types: cutting, milling, and drilling; (2) different spindle speeds: low, middle, and high; (3) different work piece materials: soft, medium, and hard; (4) different cutting depths: shallow, medium, and deep; (5) different feeding speeds: small, medium, and large; (6) different machining paths: idle, continuous cutting, periodic cutting, and non-periodic cutting.

Here, we take into consideration items (2), (3), and (6). For items (3) and (6), we choose two machining paths, which are non-periodic soft and periodic hard cutting paths. The non-periodic soft cutting condition includes a range from low to high speeds (containing the rotational speed of the spindle and the feeding speeds along the $X, Y$, and $Z$ axes). The parameters of the above two machining conditions are shown in Table 1.

\section{Measurements of Thermal Deformation and Temperature}

\subsection{Measurement system and thermal key points}

The experimental measurement setup is mainly composed of sensors, wires, and a control-and-monitor box. The sensing unit includes temperature and displacement sensors. In temperature measurement, resistance thermometers (PT100) were used to detect the variation of temperature at different thermal key points on the machine body, and eddy-current-type 
displacement sensors were used to detect the deformation due to thermal expansion at the front end of the spindle in the $X, Y$, and $Z$ directions. The thermal key points for the temperature measurements should be carefully chosen because the number of sensors and their locations significantly affect the effectiveness of compensation when building a thermal error model. Past studies ${ }^{(21,22)}$ revealed that thermal key points should be selected that (1) are close to the major heat sources, (2) have the ability to reflect the temperature field of the target system, and (3) are strongly connected to the thermal deformation. On this basis, 11 thermal key points were chosen, as listed in Table 2. Their positions are shown in Fig. 2. In addition, several eddy-current displacement sensors were pasted on the leading end of the spindle to detect displacements.

\subsection{Temperature and displacement measurements}

An integral measurement unit, including temperature and displacement sensors, was installed to synchronically retrieve readings of temperature and deformation. An experiment on thermal error modeling was performed via an integrated measurement system using the aforementioned soft and hard cutting conditions. The measured temperatures at the 11 thermal key points in the soft cutting case are shown in Fig. 3. It is revealed that, for all 11 thermal key points, temperatures vary from $25{ }^{\circ} \mathrm{C}$ (room temperature) to a maximum of $53.1{ }^{\circ} \mathrm{C}$ in the soft cutting case. Overall, the temperatures at the 11 points had an approximately linear relationship with time, with the smallest slopes for sensors T6, T3, and T2. In the hard cutting case, the temperatures varied from room temperature of $25^{\circ} \mathrm{C}$ to a maximum of $63.1{ }^{\circ} \mathrm{C}$. For each cutting condition, the temperature variations were different at different thermal key points.

The thermal drifts of the spindle nose in the $X, Y$, and $Z$ directions (respectively denoted as UX, UY, and UZ) for both cutting conditions were also obtained and are shown in Fig. 4 (soft cutting case) and Fig. 5 (hard cutting case). It is revealed that the maximum thermal drifts of the

Table 2

Locations of thermal key points.

\begin{tabular}{cc}
\hline Sensor & Location \\
\hline T1 & Environment \\
T2 & Spindle back-end \\
T3 & Spindle front-end \\
T4 & $X$-axis servo motor \\
T5 & $Y$-axis servo motor \\
T6 & $Z$-axis servo motor \\
\hline T7 & Upright column \\
T8 & Base \\
T9 & $Y$-axis ball screw \\
T10 & $X$-axis ball screw \\
T11 & $Z$-axis ball screw \\
\hline
\end{tabular}

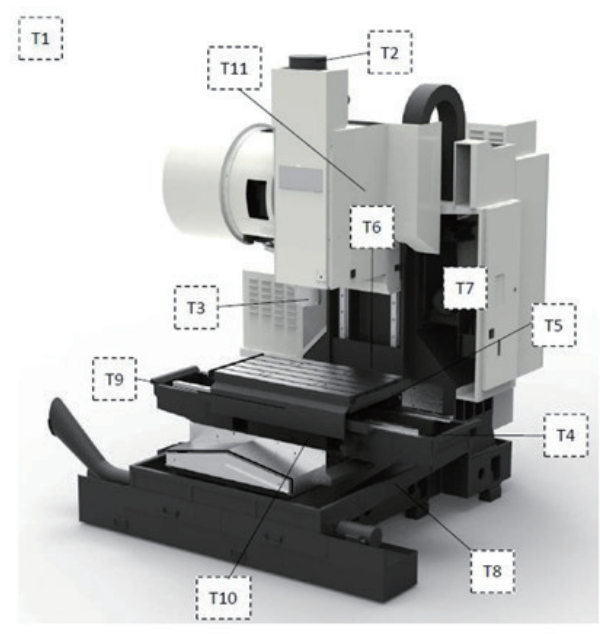

Fig. 2. Thermal key points. 


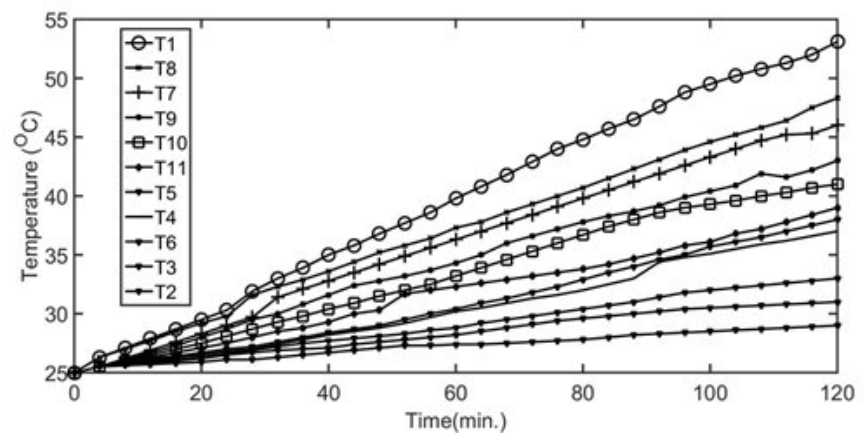

Fig. 3. Measured temperatures at thermal key points in soft cutting case.

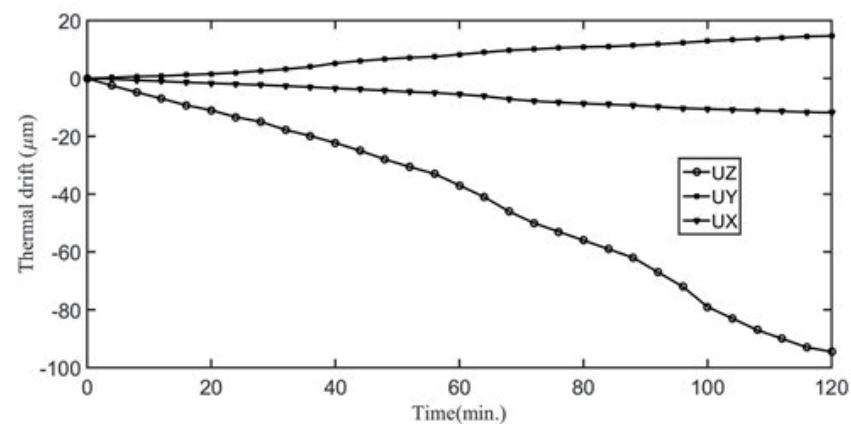

Fig. 4. Thermal drifts in non-periodic soft cutting case.

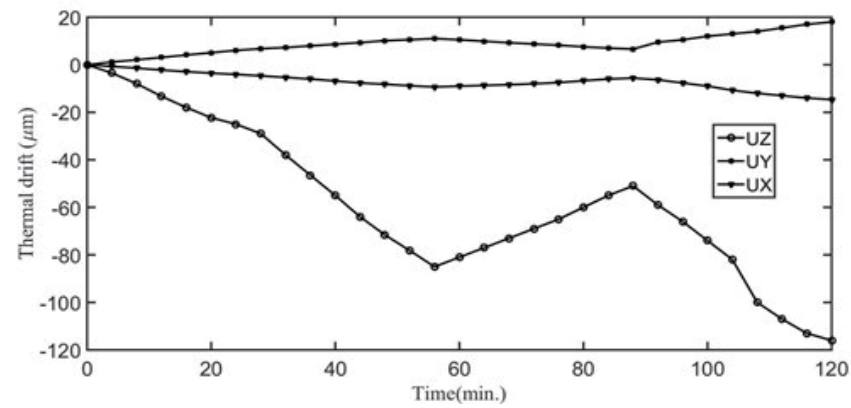

Fig. 5. Thermal drifts in periodic hard cutting case.

spindle leading end in the $X, Y$, and $Z$ directions are $+68 \mu \mathrm{m}$ (UX), $-42 \mu \mathrm{m}$ (UY), and $-113 \mu \mathrm{m}$ (UZ) in the soft cutting case and $+58 \mu \mathrm{m}$ (UX), $-35 \mu \mathrm{m}$ (UY), and $-93 \mu \mathrm{m}$ (UZ) in the hard cutting case, respectively.

\section{Mathematical Modeling of Thermal Error}

\subsection{Modeling topology}

In modeling the thermal error, we adopt a novel method based on the integrated grey system theory (IGST), which includes grey system theory (GST), in conjunction with a back- 
propagation artificial neural network (ANN) optimized by the genetic algorithm (GA) to construct the thermal error model of the target machine tool. GST is a powerful tool for analyzing a problem with spare data, which matches the nature of our thermal error modeling problem. An ANN is a supervised machine learning scheme that has high mapping ability for multiple-input and multiple-output problems, and is especially suitable for establishing nonlinear relationships between parameters. ANNs have been successfully applied in a wide range of areas, encompassing finance, personal communication, industry, and education. Furthermore, the GA is an excellent artificial intelligence method for handling optimization problems, which can be merged with an ANN to obtain optimal parameters in an IGST network. Simply speaking, the IGST scheme is the combination of the $\operatorname{GM}(1, N)$ model of GST and the back-propagation neural network (BPNN) of an ANN, whose parameters are optimized by the GA.

$\operatorname{The} \operatorname{GM}(1, N)$ model is an important part of theorems in $\operatorname{GST}^{(23)}$ and is a grey prediction model using a first-order differential equation to establish the correlation among $N$ variables. The $\operatorname{GM}(1, N)$ model is introduced as follows. First, we consider an original sequence of data. Second, we accumulate this sequence of data once. It is assumed that this accumulated data of $N$ variables will have a certain correlation obtained from a first-order differential equation. Third, we solve this first-order differential equation to find a curve to fit this accumulated data with $N$ variables.

Supposing there is an original sequence of data $x^{(0)}$ :

$$
z^{(0)}=\left\{z_{i}^{(0)} \mid i=1,2,3, \ldots, n\right\}=\left\{z_{1}^{(0)}, z_{2}^{(0)}, \ldots, z_{n}^{(0)}\right\}
$$

A first accumulation involving addition is carried out for the above original data sequence $z^{(0)}$ to obtain a new data sequence $z^{(1)}$ as follows:

$$
z^{(1)}=\left\{z_{i}^{(1)} \mid i=1,2,3, \ldots, n\right\}=\left\{z_{1}^{(0)}, \sum_{j=1}^{1} z_{j}^{(0)}, \sum_{j=1}^{2} z_{j}^{(0)}, \ldots, \sum_{j=1}^{n} z_{j}^{(0)}\right\}
$$

On the basis of the new data sequence $z^{(1)}$, we establish the following relationship between the $n$ variables in the form of a first-order differential equation:

$$
\frac{d z^{(1)}}{d t}+a z^{(1)}=U
$$

We solve the above first-order ordinary differential equation to obtain

$$
z_{t}^{*(1)}=\left(z_{1}^{(0)}-U / a\right) e^{-a(t-1)}+U / a,
$$

where $z_{t}^{*(1)}$ means the estimated value of $z_{t}^{(1)}$. Then, we perform an accumulated subtraction for 
$z_{t}^{*(1)}$. A final estimated value of $z_{t}^{(0)}$, denoted $z_{t}^{*(0)}$, is obtained, where

$$
z_{t}^{*(0)}=z_{t}^{*(1)}-z_{t-1}^{*(1)}, t=2,3, \ldots
$$

For the sake of convenience, the following variables are defined. The data sequence $z_{t}^{(0)}$ is changed to $\eta(t), z_{t}^{(1)}$ is changed to $\sigma(t)$, and $z_{t}^{*(1)}$ is changed to $\varphi(t)$.

In our investigation, we have three dependent variables of the thermal drift, denoted $y_{1}$ (UX), $y_{2}$ (UY), and $y_{3}$ (UZ), and 11 independent variables of temperatures at thermal key points, denoted $X_{1}-X_{11}$. According to the $\operatorname{GM}(1, N)$ model, we may construct the correlation between the dependent variables (e.g., $\left.y_{1}\right)$ and independent variables (e.g., $X_{1}-X_{11}$ ) based on the assumption of the first-order differential equation

$$
\frac{d \sigma_{1}}{d t}+a \sigma_{1}=b_{1} \sigma_{2}+b_{2} \sigma_{3}+\cdots+b_{11} \sigma_{12}
$$

where $\sigma_{1}=y_{1}, \sigma_{2}=X_{1}, \sigma_{3}=X_{2}, \ldots, \sigma_{12}=X_{11}$. The solution of this first-order differential equation is obtained as

$$
\sigma_{1}^{*}(t)=\left(\left(\sigma_{1}(0)-d\right)-\sigma_{1}(0) \cdot \frac{1}{1+e^{-a t}}+2 d \cdot \frac{1}{1+e^{-a t}}\right) \cdot\left(1+e^{-a t}\right),
$$

where

$$
d=\frac{b_{1}}{a} \sigma_{2}(t)+\frac{b_{2}}{a} \sigma_{3}(t)+\cdots+\frac{b_{12}}{a} \sigma_{12} .
$$

Equations (7) and (8) can be combined into the BPNN to form an IGST topology with n inputs and one output, which is illustrated in Fig. 6. There are four layers in the IGST topology, named Layer-A, Layer-B, Layer-C, and Layer-D. The input parameters are set as $\sigma_{2}(t)$, $\sigma_{3}(t), \ldots, \sigma_{12}(t)$. The connecting weights of the network are denoted by $\omega_{11}=a$ between Layer-A and Layer-B; $\omega_{21}=-y_{1}(0), \omega_{22}=2 b_{1} / a, \omega_{23}=2 b_{2} / a, \ldots$, and $\omega_{2 n}=2 b_{n-1} / a$ between Layer-B and Layer-C; and $\omega_{31}=\omega_{32}=\cdots=\omega_{3 n}=1+e^{-a t}$ between Layer-C and Layer-D. The threshold value of Layer-D is

$$
\theta(t)=\left(1+e^{-a t}\right)\left(d+y_{1}(0)\right) .
$$

Other related parameters in the layers are defined as follows:

In Layer-A (one neuron): $A=\omega_{11}$,

In Layer-B (one neuron): $B=f\left(\omega_{11} t\right)=1 /\left(1+e^{-\omega_{11} t}\right)$,

In Layer-C (13 neurons): $C_{1}=B \omega_{21}, C_{2}=\xi_{2}(t) B \omega_{22}, \ldots, C_{1 n}=\xi_{n}(t) B \omega_{2 n}(n=12)$,

In Layer-D (one neuron): $e=\omega_{31} C_{1}+\omega_{32} C_{2}+\cdots+\omega_{3 n} C_{n}-\theta(t)(n=12)$. 


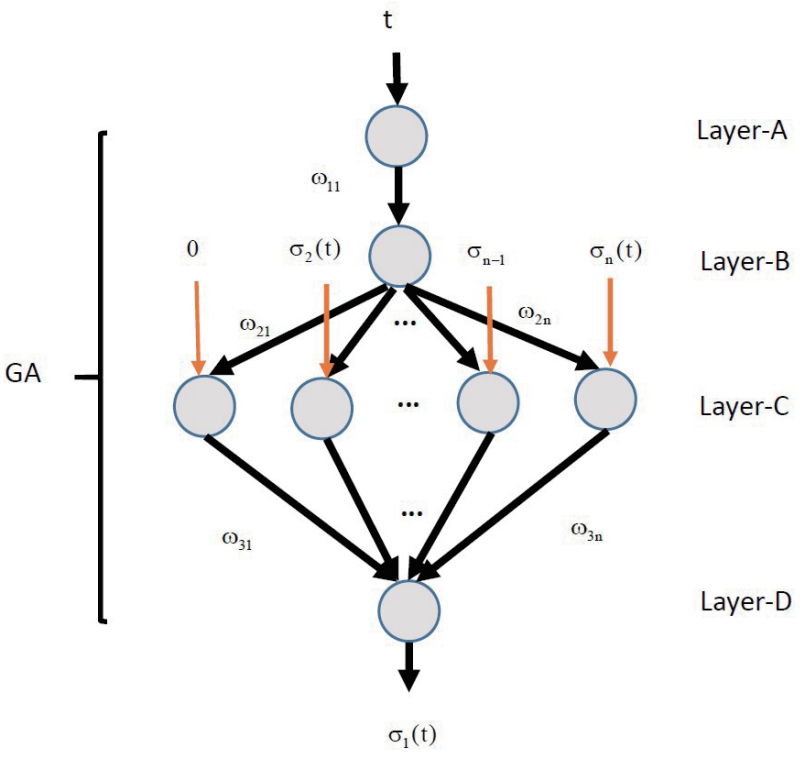

Fig. 6. (Color online) Topology of IGST.

The total error is calculated as the difference between the network output and the expected value,

$$
\delta=e-y_{1}(t)
$$

Furthermore, by using the above equations of the error $\delta$, weights $\omega$, and threshold values $\theta(t)$, the network parameters may be adjusted until convergence.

\subsection{Model building}

From measurements, we obtained 30 data pairs (taken every 4 min over a total measurement time of $120 \mathrm{~min}$ ) for both the periodic soft and non-periodic hard cutting cases to train the IGST topology. Moreover, we used the scheme of the GA in IGST to optimally search for extreme values and enhance the convergence speed of the error. It took 42 and 53 iterations to train the IGST network for the soft and hard cutting cases, respectively, to reach the error convergence criterion of $0.005 \mu \mathrm{m}$. The obtained influence weightings $\left(\left|b_{1}\right|-\left|b_{11}\right|\right)$ of the characteristic temperatures (T1-T11) on UZ, UX, and UY for the soft cutting case are shown in Figs. 7-9, respectively, and those on UZ, the most important influential thermal drift in the machine, in the hard cutting case are shown in Fig. 10. For the soft cutting case, the influences of $T 1, T 7$, $T 8$, and $T 9$ (i.e., $\left|b_{1}\right|,\left|b_{7}\right|,\left|b_{8}\right|$, and $\left|b_{9}\right|$ ) on all three thermal drifts are negligible. The other key temperatures have similar influences in the sequence

$$
\left|b_{2}\right|(T 1)>\left|b_{3}\right|(T 3)>\left|b_{6}\right|(T 6)>\left|b_{4}\right|(T 4)>\left|b_{5}\right|(T 5)>\left|b_{11}\right|(T 11)>\left|b_{10}\right|(T 10),
$$




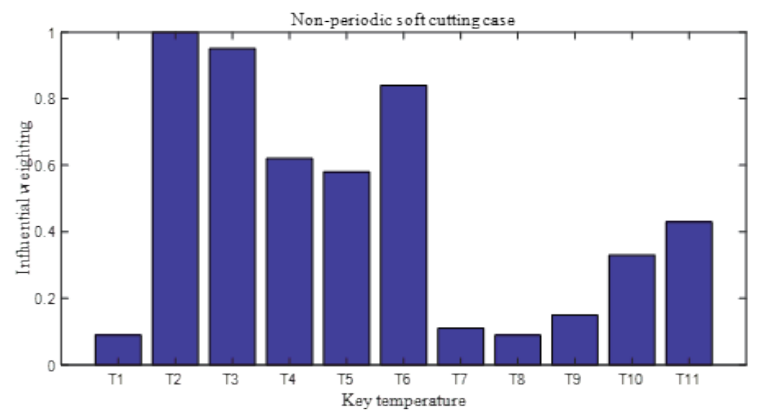

Fig. 7. (Color online) Temperature influences (weightings: $\left.\left|b_{1}\right|-\left|b_{11}\right|\right)$ at thermal key points $(T 1-T 11)$ on the thermal deformation UZ in non-periodic soft cutting case.

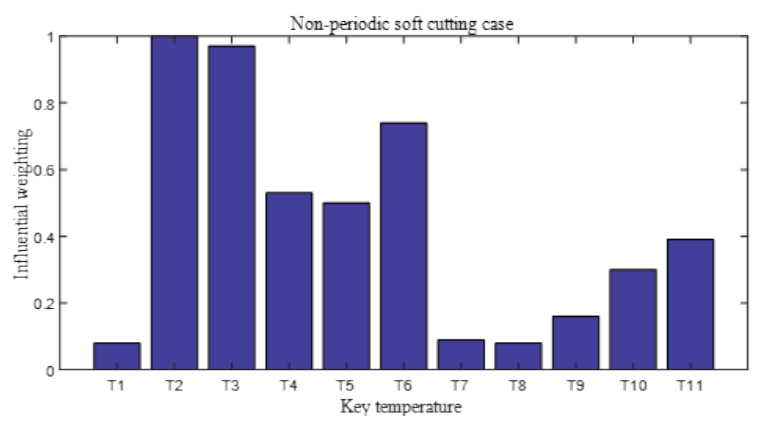

Fig. 9. (Color online) Temperature influences (weightings: $\left.\left|b_{1}\right|-\left|b_{11}\right|\right)$ at thermal key points $(T 1-T 11)$ on the thermal deformation UY in non-periodic soft cutting case.

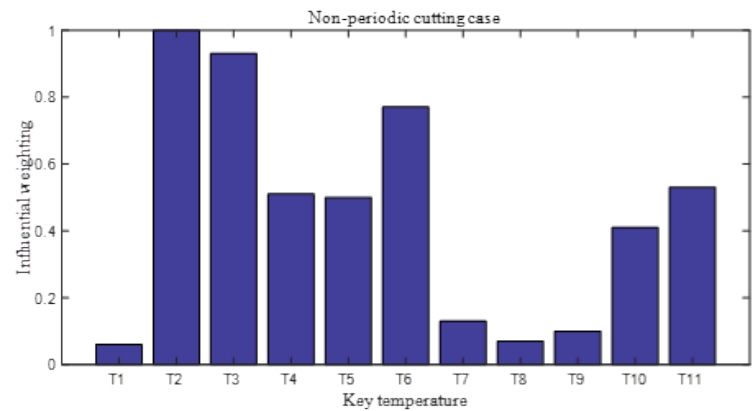

Fig. 8. (Color online) Temperature influences (weightings: $\left.\left|b_{1}\right|-\left|b_{11}\right|\right)$ at thermal key points $(T 1-T 11)$ on the thermal deformation UX in non-periodic soft cutting case.

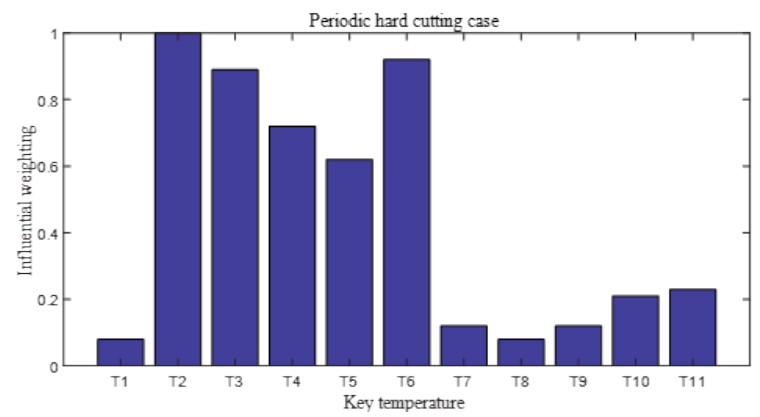

Fig. 10. (Color online) Temperature influences (weightings: $\left.\left|b_{1}\right|-\left|b_{11}\right|\right)$ at thermal key points (T1T11) on the thermal deformation UZ in periodic hard cutting case.

but have different magnitudes. On the other hand, comparing the results of the soft and hard cutting cases, it is found that the influential sequence for the hard cutting case changes to

$$
\left|b_{2}\right|(T 2)>\left|b_{6}\right|(T 6)>\left|b_{3}\right|(T 3)>\left|b_{4}\right|(T 4)>\left|b_{5}\right|(T 5)>\left|b_{11}\right|(T 11) \text {. }
$$

For both cases, it is found that five thermal key temperatures, $T 1, T 7, T 8, T 9$, and $T 10$, have negligible influence on all three thermal drifts.

Furthermore, the obtained maximal errors (relative errors) between the predicted and measured thermal drift for the soft and hard cutting cases are 0.00363 and $0.00417 \mathrm{~mm}$, respectively. Both training results are excellent, suggesting the high performance of the proposed method.

\subsection{Discussion}

To evaluate the prediction ability of the IGST topology with fixed parameters under different cutting conditions, we adopt the key temperature data from the hard cutting measurement as the 
input and apply the IGST topology and parameters obtained in the soft cutting case. In this way, we obtain a prediction result for thermal drift that has a maximal error value of $0.0891 \mathrm{~mm}$. In contrast, if we adopt the key temperature data from the soft cutting measurement as the input and apply the IGST topology and the parameters obtained in the hard cutting case, we obtain a prediction result of thermal drift that has a maximal error of $0.0929 \mathrm{~mm}$. It is found that although we used the same thermal error prediction topology for both the soft and hard cutting cases, their compensation results are different because of the different trained parameters. Using the thermal error model of IGST and its trained parameters established under the soft cutting condition to predict the thermal drift under the hard cutting condition would lead to a 22.27 times larger error $(0.929 \mathrm{~mm} / 0.00417 \mathrm{~mm})$, which is obviously unsatisfactory. Finding a universal thermal error model with fixed parameters to well predict the thermal errors for different cutting conditions is not possible here. Furthermore, if the type of machine is changed, the thermal behavior of the machine will also be different. Therefore, both the model topology as well as its parameters should be changed to obtain an optimal prediction result.

\section{Conclusions}

For tool machinery, the cutting conditions have a huge effect on the machining precision as well as on the thermal compensation results. Various cutting conditions will cause different thermal flows in a machine's body and result in a variety of temperature distributions and thermal drifts in the machine. We found that using the integrated GA-optimized grey neural network scheme as the thermal model is suitable for the thermal compensation for a C-type $\mathrm{CNC}$ machining center such as that in this study. However, under the constraint of a preset convergence criterion, the trained topology parameters of the IGST network are different for soft and hard cutting conditions. It is highly noteworthy that using the thermal error model of IGST and its trained parameters established under the soft cutting condition to predict thermal drift under the hard cutting condition would lead to a 22.27 times larger error $(0.929 \mathrm{~mm} / 0.00417 \mathrm{~mm})$. It should also be noted that a thermal error model built with fixed trained parameters under a certain cutting condition is no longer suitable when the cutting conditions are changed. In the future, more investigations will be conducted to study heat deformations and their suitable thermal compensation models for other complicated cutting conditions.

\section{Acknowledgments}

This work was carried out in the Intelligent Manufacturing Program coordinated by the Beautiful China Research Institute of Sanming University and the Operational Funding of the Advanced Talents for Scientific Research (19YG04 and 19YG05) supported by Sanming University. We also acknowledge support from Sanming University College of Mechanical and Electrical Engineering. 


\section{References}

1 J. Bryan: CIRP Ann. Manuf. Technol. 39 (1990) 645.

2 Y. Li and W. Zhao: Proc. 2012 IEEE Int. Conf. Mechatro. Auto. (2012) 2319.

3 G. Aguirre, G. PdNA, and H. Urreta: Proc. 29th Ann. Meet. Ameri. Soci. Precis. Eng. (2014) 57.

4 K. C. Wang, H. C. Shen, C. H. Yang, and H. Y. Chen: Sens. Mater. 31 (2019) 399. https://doi.org/10.18494/ SAM.2019.2104

5 Y. Li and W. Zhao: Proc. 2012 IEEE, Int. Conf. Mechatro. Auto. (2012) 2319.

6 Z. C. Du, J. G. Yang, Z. Q. Yao, and B. Y. Xue: J. Mater. Process. Technol. 129 (2002) 619. https://doi. org/10.1016/S0924-0136(02)00668-4.

7 V. P. Raja, S. R. Babu, D. V. Krishna, J. Kanchana, and P. R. Thyla: Int. J. Mech. Mechatro. Eng. 14 (2014) 77. https://doi.org/10.1007/s00170-014-5916-2.

8 S. R. Babu, V. P. Raja, J. Kanchana, and D. V. Krishna: Int. J. Eng. Technol. 3 (2014) 113. https://doi. org/10.14419/ijet.v3i2.2012

9 K. C. Wang, H. C. Shen, C. H. Yang, and H. Y. Chen: Sens. Mater. 30 (2019) 399. https://doi.org/10.4304/ jcp.9.11.2616-2620

10 Z. Feng and G. Yin: J. Comp. 9 (2014) 2616.

11 H. Liu, E. Miao, L. Zhang, L. Li. Y. Hou, and D. Tang: IEEE Access 8 (2020) 63497. https://doi.org/10.1109/ access.2020.2983471

12 K. C. Wang and P. C. Tseng: J. Adv. Mech. Des. Sys. Manuf. 4 (2010) 516.

13 K. C. Wang: J. Grey Syst. 4 (2010) 353. https://doi.org/10.6138/JIT.2014.15.7.16

14 M. Fujishima, K. Narimatsu, N. Irino, and Y. Ido: CIRP J. Manuf. Sci. Technol. 22 (2018) 111. https://doi. org/10.1016/j.cirpj.2018.04.003

15 H. Liu, E. Miao, Z. Xindong, X. Zhuang, and X. Wei: Precis. Eng. 51 (2018) 169. https://doi.org/10.1016/j. precision.2017.08.007

16 S. N. Grama, A. Mathur, and A. N. Badhe: Int. J. Mach. Tools Manuf. 132 (2018) 3. https://doi.org/10.1016/ j.ijmachtools.2018.04.004

17 P. Blaser, F. Pavlicek, K. Mori, J. Mayr, S. Weikert, and K. Wegener: J. Manuf. Syst. 44 (2017) 302. https://doi. org/10.1016/j.jmsy.2017.04.011

18 J. Mayra, J. Jedrzejewskic, E. Uhlmann, M. A. Donmez, W. Knapp, F. Hartig, K. Wendt, T. Moriwaki, P. Shore, R. Schmitt, C. Brecher, and T. Wurz, K. Wegener: CIRP Ann. Manuf. Technol. 2 (2012) 1. https://doi.org/10.1016/ j.cirp. 2012.05 .008

19 A. M. Abdulshahed, A. P. Longstaff, S. Fletcher, and A. Potdar: J. Manuf. Syst. 41 (2016) 130. https://doi. org/10.1016/j.jmsy.2016.08.006

20 M. Mares and O. Horejs: Int. J. Auto. Tech. 14 (2020) 475. https://doi.org/doi:10.20965/ijat.2020.p0475

21 M. Gebhardt, J. Mayr, N. Furrer, T. Widmer, S. Weikert, and W. Knapp: CIRP Ann. Manuf. Technol. 63 (2014) 509. https://doi.org/10.1016/j.cirp.2014.03.029

22 E. Miao, Y. Liu, H. Liu, Z. Gao, and W. Li: In. J. Mach. Tools Manuf. 97 (2015) 50. https://doi.org/10.1016/ j.ijmachtools.2015.07.004

23 J. Deng: J. Grey Syst. 1 (1989) 1. 